\title{
A Comparison of the Unpressurized Rover and Small Pressurized Rover During a Desert Field Evaluation
}

\author{
Harry Litaker, Jr. ${ }^{1}$, Shelby Thompson ${ }^{1}$, and Robert Howard, Jr. ${ }^{2}$ \\ ${ }^{1}$ Lockheed Martin, JSC, Houston, TX \\ ${ }^{2}$ Johnson Space Center, NASA
}

\begin{abstract}
To effectively explore the lunar surface, astronauts will need a transportation vehicle which can traverse all types of terrain. Currently, the National Aeronautics and Space Administration's (NASA) is investigating two lunar rover configurations to meet such a requirement. Under the Lunar Electric Rover (LER) project, a comparison study between the unpressurized rover (UPR) and the small pressurized rover (SPR) was conducted at the Black Point Lava Flow in Arizona. The objective of the study was to obtain human-in-theloop performance data on the vehicles with respect to human-machine interfaces, vehicle impacts on crew productivity, and scientific observations. Four male participants took part in four, one-day field tests using the exact same terrain and scientific sites for an accurate comparison between vehicle configurations. Subjective data was collected using several human factors performance measures. Results indicate either vehicle configuration was generally acceptable for a lunar mission; however, the SPR configuration was preferred over the UPR configuration priminarly for the SPR's ability to cause less fatigue and enabling greater crew productivity.
\end{abstract}

\section{INTRODUCTION}

For astronauts to effectively explore any planetary surface, a transport vehicle will be required. The Lunar Roving Vehicle (LRV) of the Apollo era gave investigators their first insight into the design and performance of such a surface transport vehicle in a lunar gravitational environment. Today, with NASA wanting to accomplish long-duration stays of six months, a new design will need to be developed with significantly greater performance capabilities. To address this need, NASA's Constellation Lunar Architecture Team (CxAT_Lunar) and the Constellation Lunar Surface Systems (LSS) Project are conducting studies of two configurations of planetary surface vehicles. Under the Lunar Electric Rover (LER) umbrella, the two vehicle configurations are the unpressurized rover (UPR) and the small pressurized rover (SPR).

The goal of this evaluation was to compare the feasibility and operational characteristics of both the UPR and SPR concepts in several areas such as consumables usages, EVA frequency, human-machine, and machine-machine interfaces. Such data is sought after by several programs for use in models to determine which vehicle will best fulfill the CxAT requirements.

\section{METHODS}

\section{Participants}

Four participants (all males) took part in four, one-day evaluations. Two were flight experienced astronauts and two were professional geologists. Each was experienced with the current UPR and SPR configurations with several weeks of training prior to the field trial. In addition, all participants had familiarization training on all internal and external system operations. In preparation, they took part in dry runs at the Johnson Space Center (JSC) Rock Yard.

\section{Test Environment}

The test environment for the field trials occurred at the Black Point Lava Flow; approximately 40 miles north of Flagstaff, Arizona. This test site has a wide variety of geologically relevant surface features that presented many opportunities to evaluate human performance with the Intravehicular Activities (IVA) and Extravehicular Activities (EVA) science/exploration capabilities of both vehicles. Surface characteristics included slopes with an approximate $6^{\circ}$ of vertical from top to bottom, soil mechanics from lose grain to hard-packed, surface properties from flat/smooth to rocky, and some minimal vegetation.

\section{Equipment}

Both vehicles tested during the desert field trials were medium to high fidelity functional vehicles. The UPR is a 12-wheeled, truck-style vehicle with two turrets that allows two suited crew members to rotate $360^{\circ}$ enabling them to pivot without repositioning their body to align themselves in the direction of vehicle motion or any other direction of interest (see Figure 1). Steering of the vehicle is independent so that either crew member can drive. The suspension system can be raised, allowing the vehicle to maneuver over obstacles or lowered to completely rest on the ground, allowing for the crew to disembark easily.

By contrast, the SPR has a pressurized carbon fiber and fiberglass shell that provides the crew a safe haven from the hazardous environment of the lunar surface. It provides a living area for multiple day missions away from the outpost, and a rapid EVA deployment system for scientific exploration of the lunar surface (see Figure 2). It has two operational driving stations with wireless computer displays for navigation, Global Positioning System (GPS) functionality, and vehicle system control. Located in the rear of the vehicle, 
are two functional suitports with EVA suits that are used for lunar exploration.

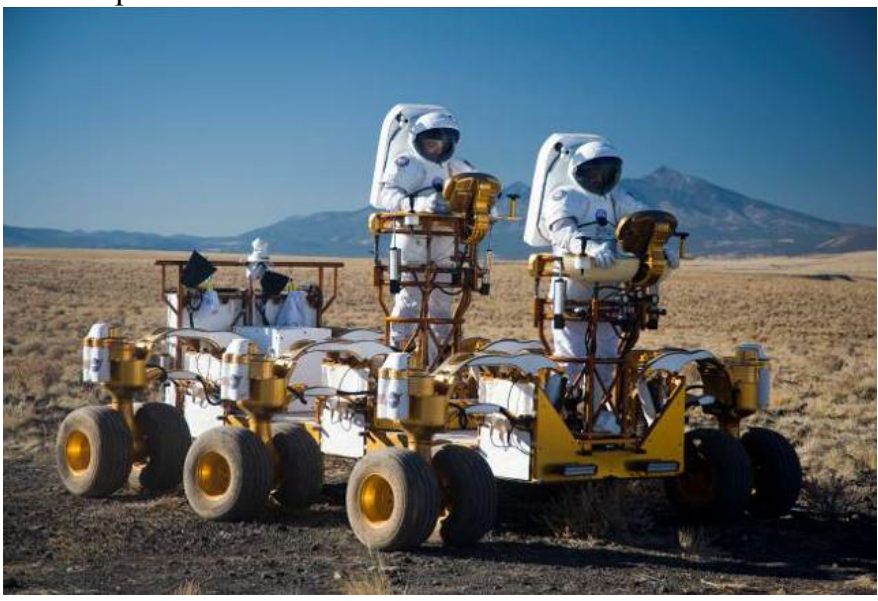

Figure 1. The UPR configuration used during the desert trials.

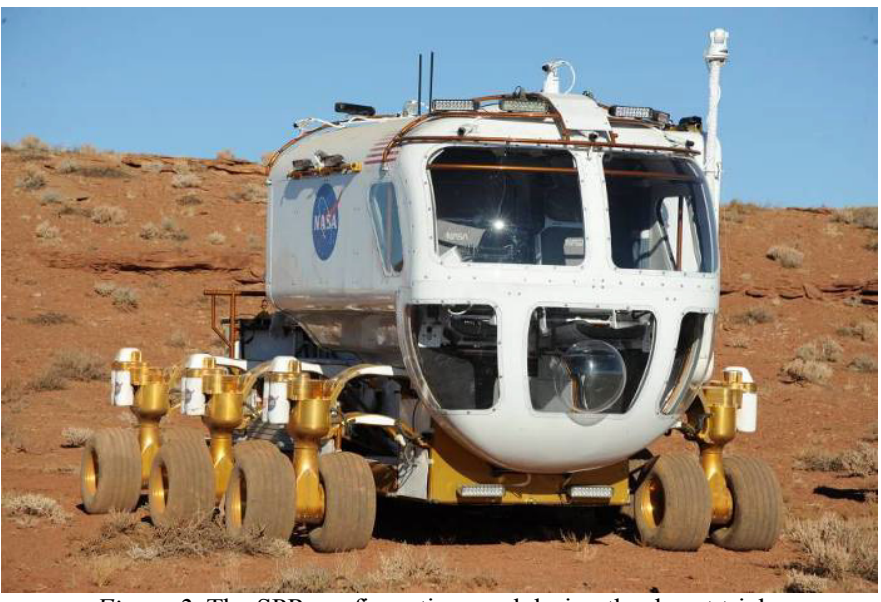

Figure 2. The SPR configuration used during the desert trials.

Participants wore the unpressurized Global Effects Mark III mockup EVA suit (see Figure 3). The mockup suit consists of a hard torso and hips with soft arms and legs. Each suit sports a mockup Portable Life Support System (PLSS) which is equipped with a fan to circulate air, communications equipment, GPS, and small cameras to photograph both still and motion images. The participants were supported by engineers from the JSC Space Suit and Crew Survival System Branch (EC5).

\section{Procedures}

A series of four predefined day missions for exploration/mapping/geological traverses, of eight hours each, were performed for both the two UPR missions and the two SPR missions. The plans were developed to identify and prioritize specific sites of scientific interest at the test region using remote sensing data that is of equivalent resolution to that expected of a crewed lunar mission without preceding robotic or crewed missions to that site. The plan included detailed timelines and traverse stations each with specific tasks associated with the scientific objectives at those stations.
Two crews of two participants ( 1 astronaut and 1 geologist) each performed all of the planned traverses, one day for each crew, and traverses using the functional requirements developed during the Lunar Architecture Team Phase 2 (LAT2) project. The Usability Testing and Analysis Facility (UTAF) collected human performance data while crew members performed the predefined scientific tasks, operated the vehicles, interfaced with vehicle systems, and performed EVAs. A combination of human factors metrics [i.e., modified Cooper-Harper Handling Quality Rating Scales for Driving and Display/Control, the Borg Scale for Rating Perceived Exertion (RPE), the Corlett/Bishop Discomfort Scale, and a Fatigue Scale] and customized post-questionnaires were used to evaluate and compare human performance between the UPR and SPR.

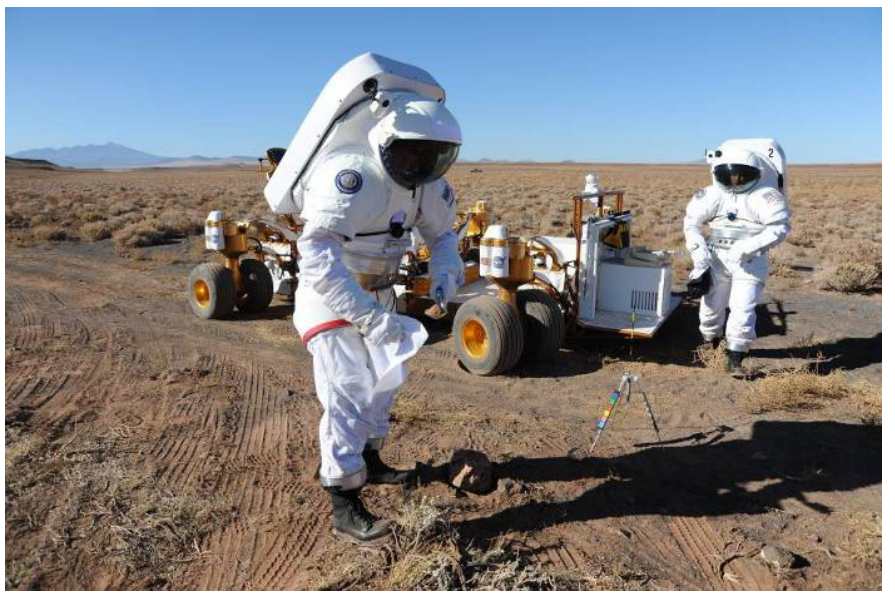

Figure 3. Participants in the Global Effects Mark III mockup EVA suits.

Data was recorded by the investigator approximately every 30 to 45 minutes during the day, as well as field notes being taken of all planned and unplanned traverse activities.

\section{RESULTS}

For the comparative analysis, the missions for both vehicle configurations were the same in all respects. The major areas of comparative interests were: driving characteristics, visibility characteristics, display and control (D\&C), seating, and EVA. The Cooper-Harper was interpreted using dot-plots, where the dots themselves denote the rating given and the size of the dot corresponds to the number of scores at that rating. Given the small sample size, subjective data from the rating scales and post-questionnaires was analyzed using the nonparametric Wilcoxon Signed Ranks Test to evaluate differences between paired scores across both vehicles. All post-evaluation questions were measured on a 10-point scale with higher values being less desirable in terms of acceptability. Overall averages for both the UPR and SPR are shown in Table 1. Discomfort was measured on an 11-point scale $(0-10)$, RPE on a 15 -point scale $(6-20)$, and Fatigue on a 10 -point scale $(1-10)$.

\section{Ratings for Driving}


The driving terrain varied from flat/smooth to ruddy with steep rocky grades during the day's mission. There is a greater amount of variation shown in the Cooper-Harper scores for driving with the UPR as compared to the SPR - scores ranged from 3 to 6 for the UPR, while the SPR gathered only $3 \mathrm{~s}$ (see Figure 4). The frequency of scores for the UPR suggests that most driving performance required moderate to minimal compensation; however, there were some points during the mission where obtaining adequate driving performance required the operator to use considerable to extensive compensation. Examination by terrain showed no effect. In contrast, scores for the SPR suggests that driving performance was constant at a desirable level with minimal compensation needed throughout. Field notes concur with this finding.

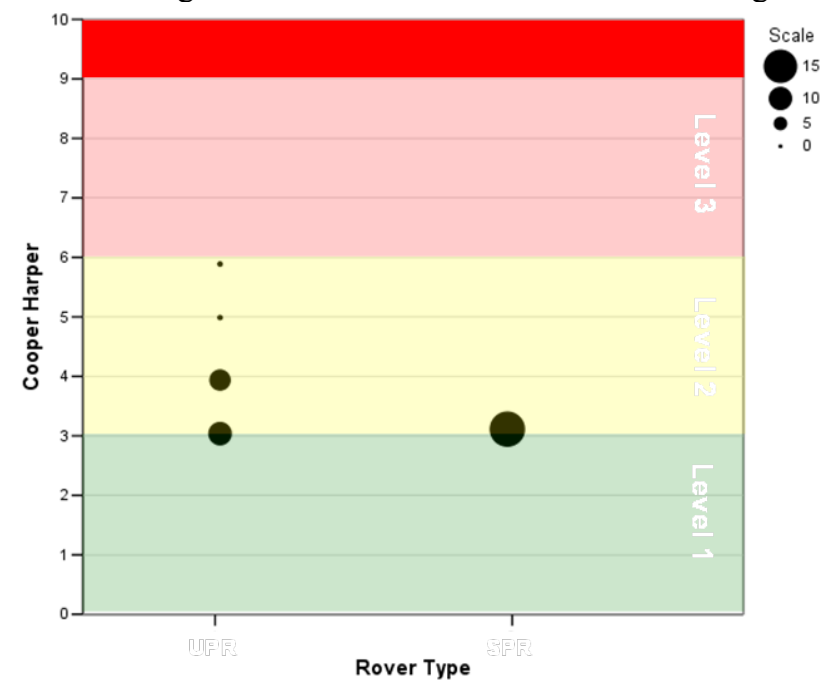

Figure 4. Cooper-Harper ratings for driving by rover type for all four days of testing.

Participants rated twelve characteristics of driving on the postquestionnaire. Results from the Wilcoxon test indicated a significant difference for driving ratings, $z=-2.38, p=.017$ with the mean of the ranks in favor of the SPR being 5.31, while the mean of the ranks in favor of the UPR was 2.50. This indicates that participants found the SPR configuration easier to drive than the UPR over the same type of environment.

Table 1.

Average Task Scores for the SPR and UPR

\begin{tabular}{lll}
\hline Tasks & UPR & SPR \\
\hline Driving & 3.51 & 3.17 \\
Displays and Controls & 3.88 & 4.05 \\
Visability & 3.15 & 3.22 \\
Seating & 3.04 & 2.82 \\
EVA Factors & 3.58 & 4.13 \\
\hline
\end{tabular}

\section{Ratings for Displays and Controls}

The Cooper-Harper ratings for displays and controls (D\&C) revealed a variation in scores for both configurations.
However, the UPR scores fell mostly about a rating of 4 while for the SRP a 3 (see Figure 5). The scores for the UPR suggest that while performance was acceptable, there were annoying deficiencies that required moderate user compensation. In addition, a single Cooper-Harper rating of 10 was recorded for the UPR due to the front turret spinning uncontrollably because of an uncommanded software glitch.

For the SPR configuration, the majority of the scores are clustered around 3 with some variance resulting in a max score of 5. This suggests that while performance was acceptable when interfacing with the D\&Cs, annoying deficiencies required the operator to use moderate compensation. Therefore, improvements are suggested to increase performance with the D\&C human-machine interfaces.

Sixteen different characteristics of the $\mathrm{D} \& \mathrm{C}$ were rated for both vehicle configurations. Wilcoxon analysis found no significant difference for the rating of the $\mathrm{D} \& \mathrm{C}$ for the two rover configurations, $z=-.320, p=.749$. Issues reported with both $\mathrm{D} \& \mathrm{C}$ vehicle configurations was loss of navigational pages, button size, touch screen responsiveness, sun glare, and the need to stop the vehicle to input or change navigational waypoints due to vehicle motion. Dust on the display screen was an issue with readability on the UPR, while reaching the display screen and vibration was an issue with the SPR configuration.

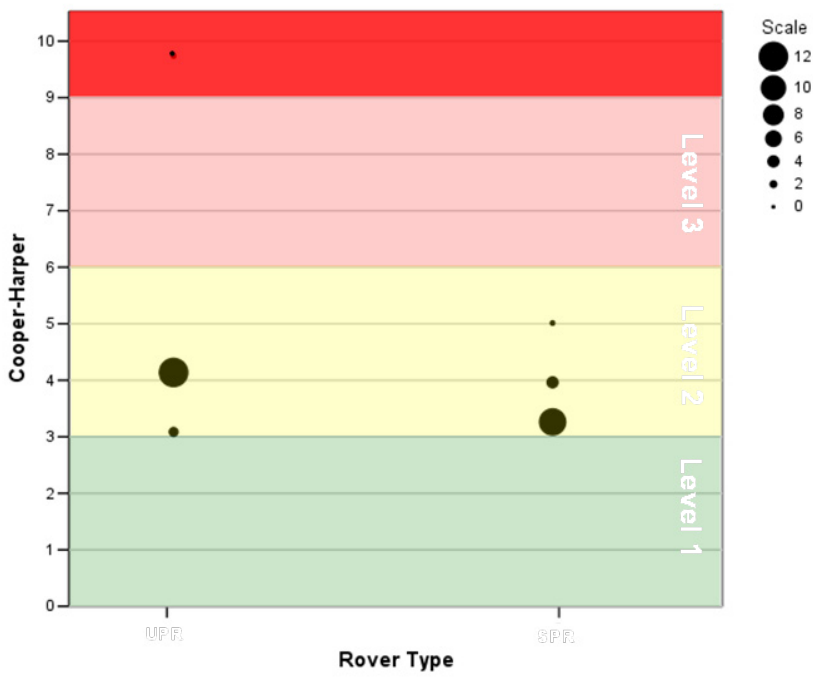

Figure 5. Cooper-Harper ratings for the displays and controls interface by rover type for all four days of testing.

With the controls, hand fatigue when using the joystick in the UPR was an issue. Participants noted an armrest or hand rest was needed to help minimize fatigue, especially if a pressurized suit was used. This concurs with an early evaluation of the UPR (DeSantis et al., 2008).

\section{Ratings for Visibility}

A major component to driving is visibility. There were eight characteristics classified for visibility that were rated. No 
significant difference was observed between the two vehicle configurations using the Wilcoxon Signed Ranks Test, $z=$ $.524, p=.588$.

Overall, visibility of the SPR's window configuration was reported as highly acceptable with the panoramic view of the area making the crew feel immersed in the environment. This concurs with two earlier design evaluations (Litaker et al., 2008a \& 2008b). However, viewing of the aft wheels was an issue in the SPR configuration, where by the wheels could not be seen as well as compared to the UPR configuration resulting in limited situational awareness (SA) of obstructions and wheel alignment. Similarly, SA was a problem in the UPR due to limited field-of-view capabilities within the EVA suit. However, rotating in the turret did to some extent mitigate this problem.

\section{Ratings of Vehicle Seating}

Seven factors were rated by participants for each vehicle configuration in terms of seating. A Wilcoxon analysis revealed no significant difference between the participants ratings, $z=-1.38, p=.167$. There were some negatives for both vehicles. For the UPR configuration, participants reported they would rather sit more than stand. In addition, to there needing to be arm and hand rests for driving, some type of handrails for riding within the turret was also desired.

The cockpit seats in the SPR were reported as comfortable and stable, especially in rough terrain. High marks were also given to the adjustability of seat back. However, the width between the two cockpit seats made the aisle extremely tight for a crew member to get down to the lower bubble for scientific observations (see Figure 6).

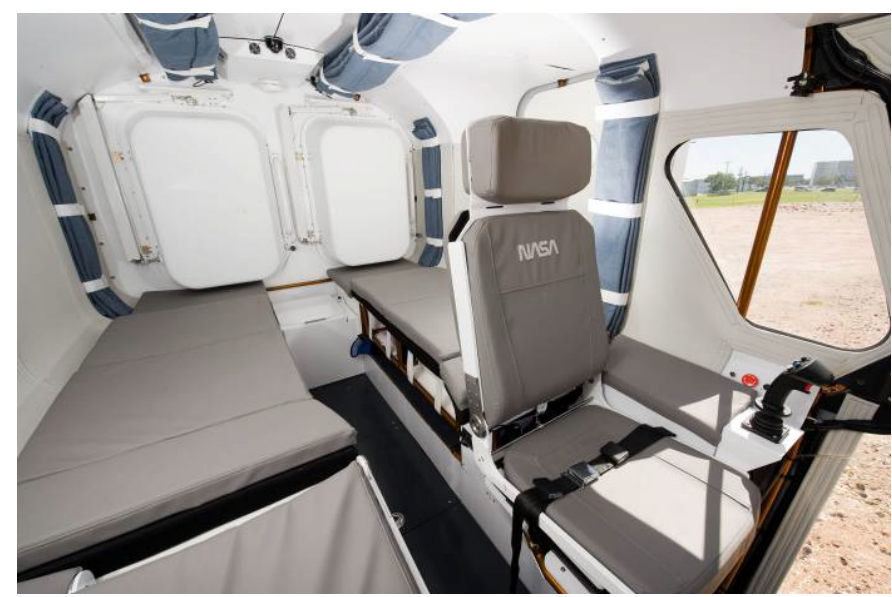

Figure 6. Interior of the SPR showing the left seat and controller. The right seat is in an incline position to illustrate the sleep station. Note the two suit ports in the back.

\section{Ratings of EVA Factors}

It is estimated that some 30,000 more hours of EVA time could be involved in lunar exploration as compared all other mission combined (i.e., International Space Station, Shuttle, and Pre-Shuttle EVA times; Gernhardt et al., 2008). With this is mind, ten EVA factors were rated by participants for both vehicle configurations. A comparison of both vehicles using the Wilcoxon test only approached significance, $z=-1.89, p=$ .058. On average the SPR was rated less positive than the UPR. This was due to the difficulty in operating the manual suit port mechanisms (see Figure 7); future designs will integrate a "flight-by-wire" approach that should solve this issue. In addition, guides will be included on future designs to aid in positioning.

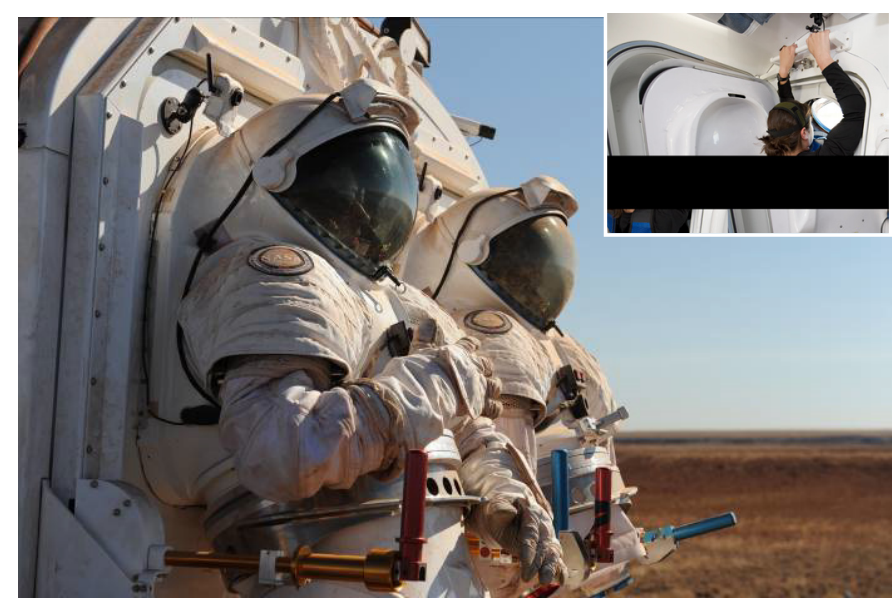

Figure 7. EVA suits locked into the suit ports on the SPR. Insert photo on right shows participant climbing through suit port into suit.

\section{Perceived Exertion, Discomfort, and Fatigue}

Given the uncertain impact of the vehicle configuration on EVA activities, the investigators examined the relationship of operational tasks and EVA activities using the Rating of Perceived Exertion (RPE), discomfort, and fatigue. Investigators collected both sets of data approximately every 45 minutes during the test session from each participant. Wilcoxon statistical analysis on the RPE data revealed no significant difference between the two vehicle configurations across the mission, $z=-.051, p=.959$. However, in terms of participant discomfort, there was a significant difference, $z=$ $2.49, p=.013$. The mean of the ranks in favor of the SPR was 6.78 , while the mean of the ranks in favor of the UPR was 2.50. There was more participant discomfort with the UPR configuration than with the SPR configuration. The areas mostly reported for discomfort included the back and shoulders for both vehicle configurations. Specific to the UPR, participants reported discomfort in the foot, ankle, and knee due to vibration of the vehicle over rough terrain and having to stand the entire time. In addition, discomfort was reported for the hand because of the absence of a hand/arm rest for the vehicle's control stick.

Ratings of fatigue were collected, before the mission day began (pre-flight) and after the completion of the mission day (post-flight), for both vehicle configurations. A plot of the ratings clearly show there to be no difference for pre-flight fatigue between the two vehicle configurations; however, post-flight ratings reveal significantly more fatigue with the 
UPR configuration than with the SPR configuration (see Figure 8).

Participants indicated several reasons for such a dramatic change in scores. Most notably, participants took full advantage of the suit ports by getting out of the suit and resting between EVAs. This helped reduce the fatigue and tended to make the crew more productive over the course of the mission. By comparison, every moment on the UPR is spent in the suit. In addition, the vibration associated with the UPR configuration added to their fatigue. Participants simply could not find a restful position in the turret and endured a continual pounding from the vehicle over rough terrain. Vibration in the SPR configuration was reported as light with only minor driving fatigue being reported.

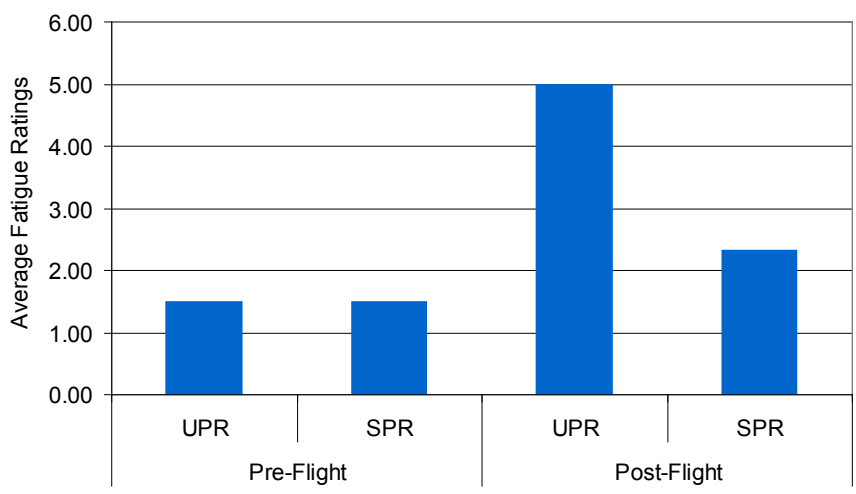

Figure 8. Participant's pre- and post-flight fatigue for both vehicle configurations.

\section{CONCLUSIONS}

Generally, participants believed either vehicle could be acceptable for a lunar mission; however, they preferred the SPR over the UPR configuration. Primary rationale for their preference included the SPR causing less fatigue and enabling greater crew productivity. The driving performance of the SPR required less compensation than the UPR according to Cooper-Harper ratings. Displays and controls for both vehicle configurations required moderate compensation with improvements to the user interface being mandatory. Suggested interface redesign centered around button size on the touch screens, less complicated menu navigation and more consistency with the Graphic User Interface (GUI).

For visibility, the refinement of side window configurations to improve lateral field-of-view for better SA is needed for the SPR, as well as, a sun shade to reduce glare. As for the UPR, a redesign of the turret placement and display position for improve visibility is needed. Issues with the suit port mechanism made attaching and disembarking problematic for the SPR. There was no significant difference for RPE scores used as a measure of crew health during EVA operations. However, there was more discomfort report for the UPR due to the constant standing and lack of mobility provided by the turrets. This resulted in higher fatigue rates reported for the UPR by the end of the mission.

These issues, and others, have been highlighted to engineers involved with the rover project. A new prototype is in the works with completion slated for summer of 2009 taking into account these findings. Another desert trial is planned for Fall of 2009 that will incorporate longer duration missions in the SPR.

\section{ACKNOWLEDGEMENTS}

We would like to recognize Dr. Michael Gernhardt for obtaining the funding for the project, Dr. Andrew Abercomby for his work on the engineering test plan, the Habitability Design Center at NASA/JSC: Rich Szabo, Carl Conlee, and Evan Twyford, for their illustrative and design work and the editing work of Dr. Victor Ingurgio of the NASA/JSC Anthropometry and Biomechanics Facility (ABF). To Regan Geeseman at NASA/JSC Information Resources Directorate (IA) for his photographic work of the vehicle used throughout this paper. Thanks also to Dr. Robert Ambrose and his team at the NASA/JSC Automation, Robotics, and Simulation Division (ER), Extravehicular Activity Office (XA), and Joe Kosmo and Barbara Roming's team with the NASA/JSC Space Suit and Crew Survival System Branch (EC5) for all their hard work in designing, building, and testing these amazing vehicles.

\section{REFERENCES}

DeSantis, L., Thompson, S., Ferrer, M.A., \& Sudhakar, R. (2008). A quick-look usability and ergonomic analysis of the Chariot lunar rover. NASA/Johnson Space Center. Internal NASA Document.

Gernhardt, M.L., Abercromby, A., Ambrose, R.O., Howard, R., Kosmo, J.J., Ney, Z., \& Roming, B. (2008). Engineering evaluation of small pressurized rover and unpressurized rover configurations during simulated planetary surface exploration. EVA Physiology, Systems \& Performance Project, NASA/Johnson Space Center. Internal NASA Document.

Litaker, Jr., H.L., Thompson, S., \& Howard, R. (2008a). Window placement evaluation for the Small Pressurized Rover (SPR) configuration 2. NASA/Johnson Space Center. Internal NASA Document.

Litaker, Jr., H.L., Howard, R., \& Ferrer, M.A. (2008b). Lunar rover habitability volume evaluation on configuration two. NASA/Johnson Space Center. Internal NASA Document. 